Supplement of Geosci. Model Dev., 12, 1991-2008, 2019

https://doi.org/10.5194/gmd-12-1991-2019-supplement

(C) Author(s) 2019. This work is distributed under

the Creative Commons Attribution 4.0 License.

(c) (1)

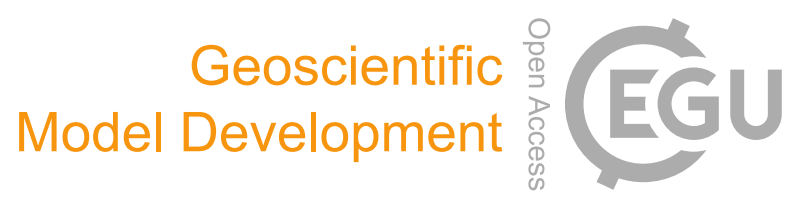

Supplement of

\title{
ATTILA 4.0: Lagrangian advective and convective transport of passive tracers within the ECHAM5/MESSy (2.53.0) chemistry-climate model
}

Sabine Brinkop and Patrick Jöckel

Correspondence to: Sabine Brinkop (Sabine.Brinkop@dlr.de)

The copyright of individual parts of the supplement might differ from the CC BY 4.0 License. 


\section{ATTILA 4.0: Lagrangian Advective and Convective Transport of Passive Tracers within the ECHAM5/MESSy (2.53.0) Chemistry Climate Model}

\section{Sabine Brinkop ${ }^{1,2}$, Patrick Jöckel ${ }^{1}$}

${ }^{1}$ Deutsches Zentrum für Luft- und Raumfahrt, Institut für Physik der Atmosphäre, Oberpfaffenhofen, 82230 Wessling, Germany

${ }^{2}$ Meteorologisches Institut der Universität München, 80333 München, Germany sabine.brinkop@dlr.de,patrick.joeckel@dlr.de

This manual is part of the electronic supplement of our article "ATTILA 4.0: Lagrangian Advective and Convective Transport of Passive Tracers within the ECHAM5/MESSy (2.53.0) Chemistry Climate Model" in Geosci. Model Dev. (2019), available at: http://www.geosci-model-dev.net 


\section{Contents}

1 Overview $\quad 3$

2 Infrastructure submodel TRANSFORM (messy_main_transform_bi.f90) 3

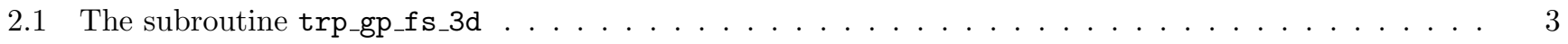

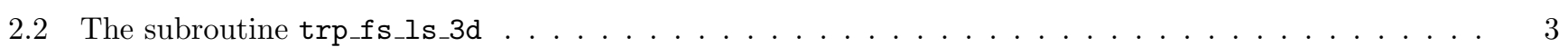

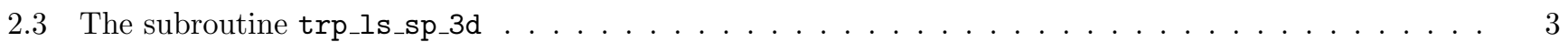

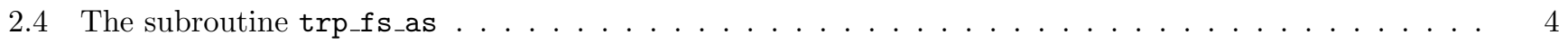

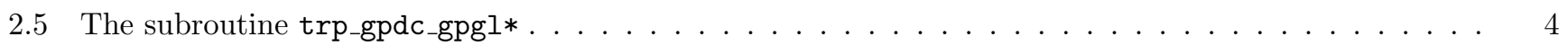

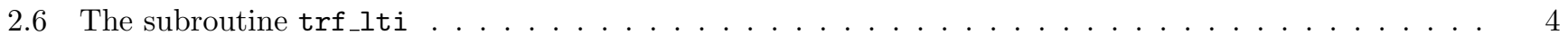

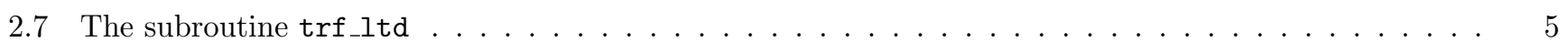

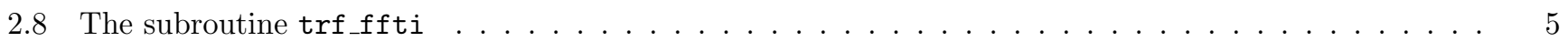

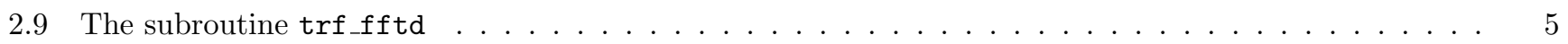

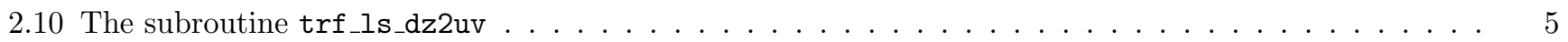

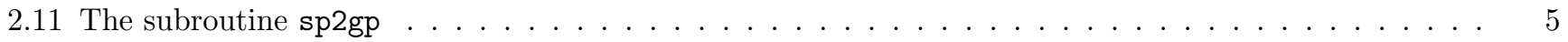

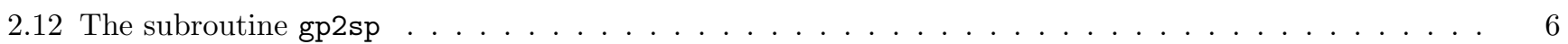

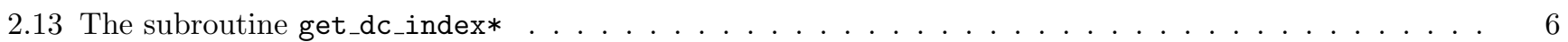

2.14 The subroutine scatter_glix_1d $\ldots \ldots \ldots \ldots \ldots \ldots \ldots \ldots \ldots \ldots \ldots \ldots \ldots \ldots \ldots \ldots \ldots$

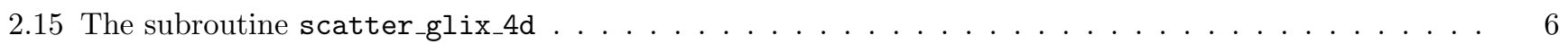

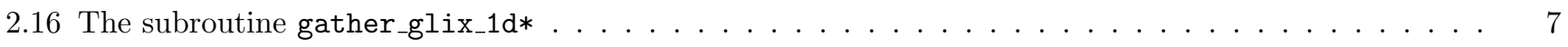

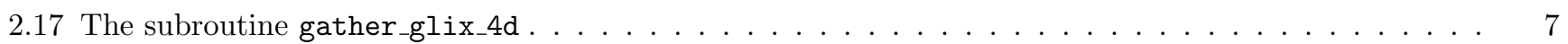

3 Sub-submodel ATTILA_TOOLS (messy_attila_tools_e5) $\quad 7$

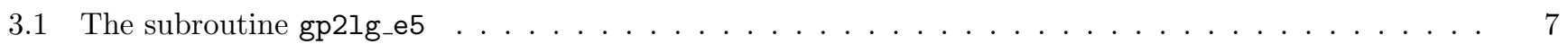

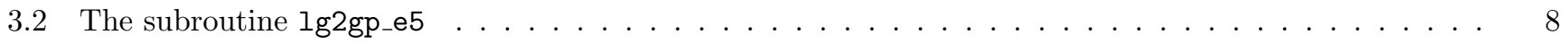

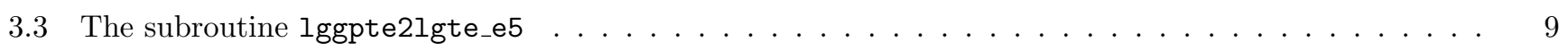

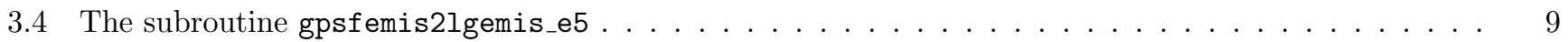

4 ATTILA namelists $\quad 9$

5 LGGP namelist $\quad 13$

6 LGVFLUX namelist $r$

$\begin{array}{llr}7 & \text { LGTMIX namelist } & 15\end{array}$

8 DRADON namelist extension $\quad 16$

$\begin{array}{lr}\text { References } & 17\end{array}$ 


\section{Overview}

This supplement describes in detail the technical infrastructure of new and extended submodels (submodel TRANSFORM, sub-submodel ATTILA_TOOLS), and the namelists of the new diagnostic submodels (LGGP, LGVFLUX) and extended or new physical submodels (LGTMIX, DRADON, ATTILA).

\section{Infrastructure submodel TRANSFORM (messy_main_transform_bi.f90)}

This module contains subroutines for the transposition and transformation of variables (i.e., Fortran arrays) between various representations, such as spectral, grid-point and Lagrangian. Subroutines marked with an asterisk are used specifically within ATTILA.

\subsection{The subroutine trp_gp_fs_3d}

\begin{tabular}{|c|c|c|c|}
\hline SUBROUTINE & trp_gp_fs_3d & (sign, & $f s)$ \\
\hline name & type & intent & description \\
\hline \multicolumn{4}{|c|}{ mandatory arguments: } \\
\hline sign & INTEGER, & IN & $\begin{array}{l}\operatorname{sign}=1: G \mathrm{GP}->\mathrm{FS} \\
\text { sign }=-1: \mathrm{GP}<-\mathrm{FS}\end{array}$ \\
\hline pgp & REAL(DP), DIMENSION(:,:,:) & INOUT & grid-point space $3 \mathrm{~d}$ (GP) \\
\hline pfs & REAL(DP), DIMENSION(:,:::) & INOUT & Fourier space (FS) \\
\hline
\end{tabular}

This subroutine transposes data from grid-point space in parallel domain decomposition into Fourier space ( $\operatorname{sign=1}$ ) in parallel decomposition and vice versa ( $\operatorname{sign}=-1)$.

\subsection{The subroutine trp_fs_ls_3d}

\begin{tabular}{|c|c|c|c|}
\hline \multicolumn{2}{|c|}{ SUBROUTINE trp_fs_ls_3d } & \multicolumn{2}{|c|}{ (sign, fs, ls) } \\
\hline name & type & intent & description \\
\hline \multicolumn{4}{|c|}{ mandatory arguments: } \\
\hline sign & INTEGER, & IN & $\begin{array}{l}\text { sign }=1: \text { FS }->\text { LS } \\
\text { sign }=-1: \text { FS }<- \text { LS }\end{array}$ \\
\hline fs & REAL(DP), DIMENSION $(:,:,:)$ & INOUT & Fourier space (FS) \\
\hline ls & REAL(DP), DIMENSION $(:,:,:)$ & INOUT & Legendre space (LS) \\
\hline
\end{tabular}

This subroutine transposes data from Fourier space in parallel decomposition into Legendre space (sign=1) in parallel decomposition and vice versa ( sign=-1).

\subsection{The subroutine trp_ls_sp_3d}

\begin{tabular}{|llll|}
\hline \multicolumn{2}{|l|}{ SUBROUTINE } & trp_ls_sp_3d & \multicolumn{2}{l|}{ ( $\mathrm{sign}, \mathrm{pls}, \mathrm{psp})$} \\
\hline name & type & intent & description \\
\hline mandatory & arguments: & & \\
sign & INTEGER, & IN & sign=1: LS $->$ SP \\
& & & sign=-1: LS $<-$ SP \\
pls & REAL(DP), DIMENSION $(:,:,:)$ & INOUT & Legrendre space (LS) \\
psp & REAL(DP), DIMENSION $(:,:,:)$ & INOUT & Spectral space (SP) \\
\hline
\end{tabular}

This subroutine transposes data from Legendre space in parallel decomposition into spectral space ( ign=1) in parallel decomposition and vice versa $(\operatorname{sign}=-1)$. 


\subsection{The subroutine trp_fs_as}

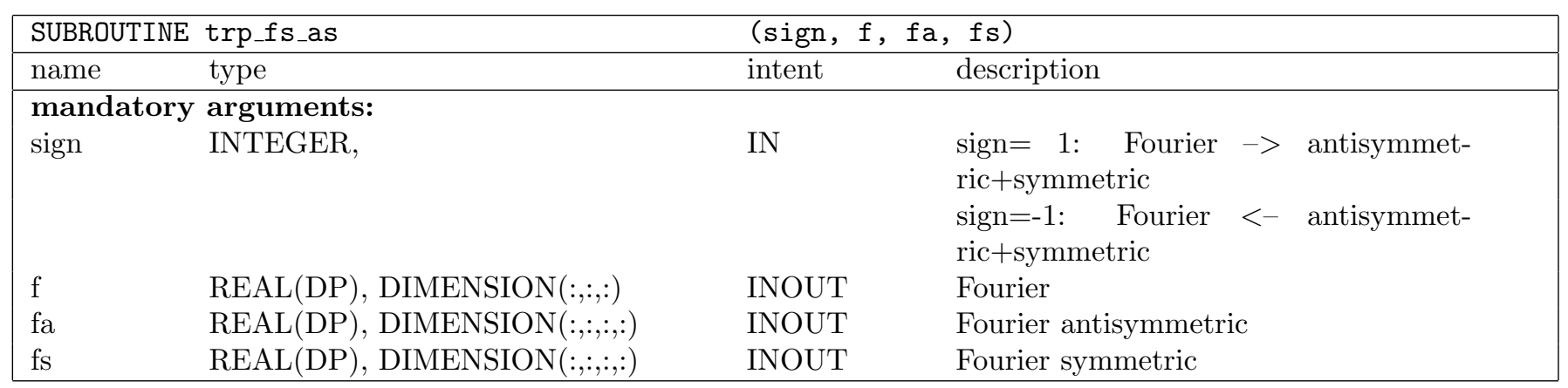

This subroutine transposes data from Fourier space in parallel decomposition into the corresponding antisymmetric

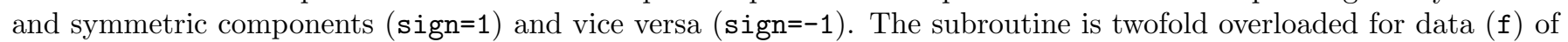
rank 2 and 3 (as shown here), respectively.

\subsection{The subroutine trp_gpdc_gpgl*}

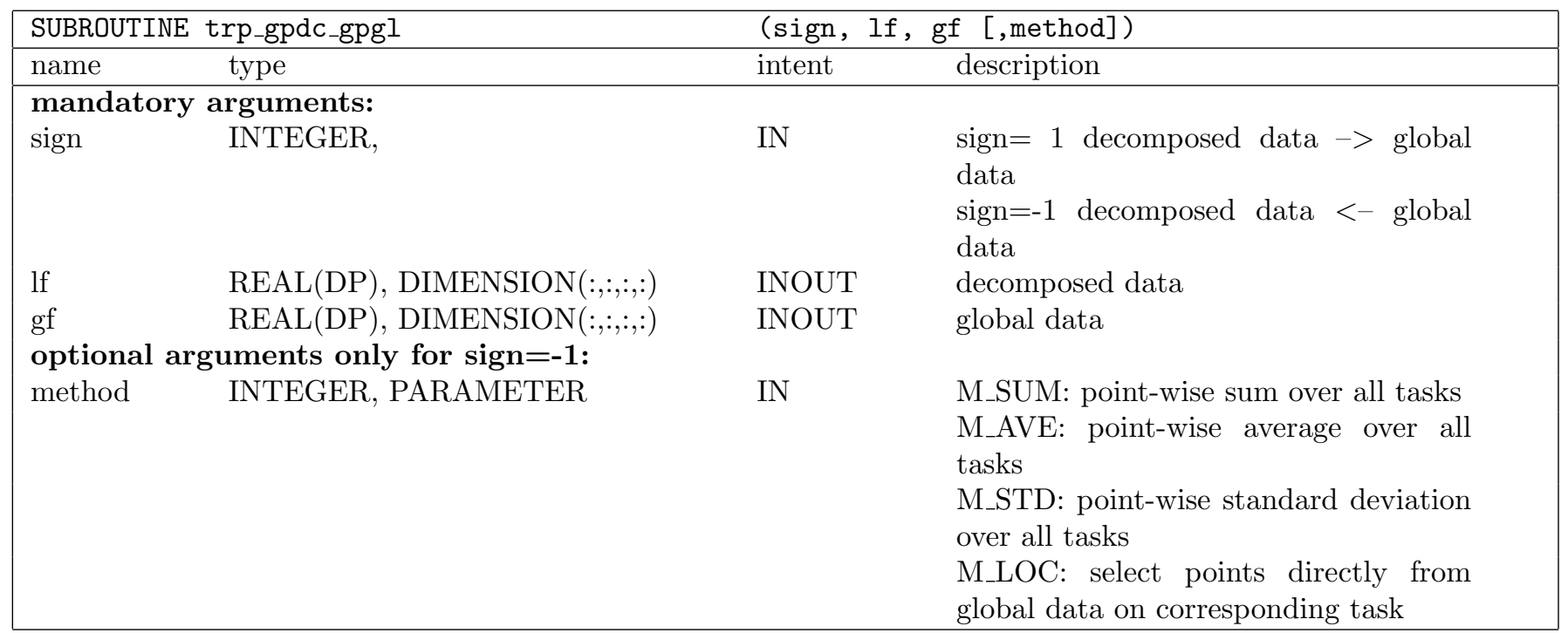

This subroutine is for the global domain cloning in grid-point space. It transposes decomposed grid-point data from parallel domain decomposition into global data for each task (sign=1) and vice versa $($ sign=-1). For the reduction from the $n$ (number of tasks) global arrays into a joined decomposed data array, four methods are selectable: M_SUM $=0$, M_AVE $=1$, M_STD $=2$, M_LOC $=3$. The subroutine is threefold overloaded for data arrays of rank 2,3 and 4 (as shown above), respectively.

\subsection{The subroutine trf_lti}

\begin{tabular}{|llll|}
\hline \multicolumn{2}{|l|}{ SUBROUTINE } & trf_lti & \multicolumn{2}{l|}{ (fa, fs, $\mathrm{ls}$ ) } \\
\hline name & type & intent & description \\
\hline mandatory & arguments: & & \\
fa & REAL(DP), DIMENSION $(:,:,:,:)$ & OUT & anti-symmetric \\
fs & REAL(DP), DIMENSION $(:,:,:,:)$ & OUT & symmetric \\
ls & REAL(DP), DIMENSION $(:,:,:)$ & IN & \\
\hline
\end{tabular}

This subroutine calculates the inverse Legendre transformation in parallel decomposition and returns the anti-symmetric and the symmetric Fourier coefficients. This subroutine is threefold overloaded for arrays (1s) of rank 1, 2, and 3 (as shown above), respectively. 


\subsection{The subroutine trf_ltd}

\begin{tabular}{|llll|}
\hline \multicolumn{2}{|l|}{ SUBROUTINE } & trf_lti & \multicolumn{2}{l|}{ ( $\mathrm{s}, \mathrm{fa}, \mathrm{fs})$} \\
\hline name & type & intent & description \\
\hline mandatory & arguments: & \\
ls & REAL(DP), DIMENSION $(:,:,:)$ & OUT & \\
fa & REAL(DP), DIMENSION $(:,:,:,:)$ & IN & anti-symmetric \\
fs & REAL(DP), DIMENSION $(:,:,:,:)$ & IN & symmetric \\
\hline
\end{tabular}

This subroutine calculates the (direct) Legendre transformation in parallel decomposition from the anti-symmetric and the symmetric Fourier coefficients. This subroutine is threefold overloaded for arrays (1s) of rank 1, 2, and 3 (as shown above), respectively.

\subsection{The subroutine trf_ffti}

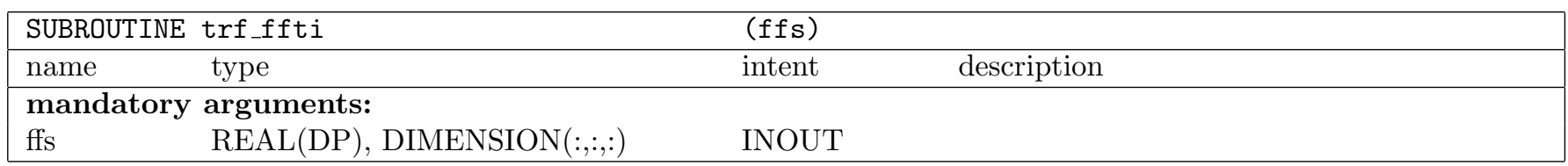

This subroutine calculates the inverse fast Fourier transformation.

\subsection{The subroutine trf_fftd}

\begin{tabular}{|c|c|c|c|}
\hline SUBROUTINE & trf_ffti & $(f f s)$ & \\
\hline name & type & intent & description \\
\hline $\begin{array}{l}\text { mandatory } \\
\text { ffs }\end{array}$ & $\begin{array}{l}\text { arguments: } \\
\text { REAL(DP), DIMENSION }(:,:,:)\end{array}$ & INOUT & \\
\hline
\end{tabular}

This subroutine calculates the direct fast Fourier transformation.

\subsection{The subroutine trf_ls_dz2uv}

\begin{tabular}{|c|c|c|c|}
\hline SUBROUTINE & $\operatorname{trf} \_$ls_dz2uv & $(d, z$ & \\
\hline name & type & intent & description \\
\hline mandatory & arguments: & & \\
\hline $\mathrm{d}$ & REAL(DP), DIMENSION(:,:,:) & IN & divergence \\
\hline $\mathrm{z}$ & REAL(DP), DIMENSION(:,:,:) & IN & vorticity \\
\hline $\mathrm{u}$ & REAL(DP), DIMENSION(:,:,:) & OUT & $\mathrm{u} * \cos ($ lat $)$ \\
\hline $\mathrm{v}$ & REAL(DP), DIMENSION(:,:,:) & OUT & $\mathrm{v} * \cos ($ lat $)$ \\
\hline
\end{tabular}

This subroutine calculates the wind vector components $u \cdot \cos ($ lat $)$ and $v \cdot \cos ($ lat $)$ by transformation from divergence and vorticity data in Legendre space. This subroutine is twofold overloaded for arrays of 2 and three (as shown above), respectively.

\subsection{The subroutine sp2gp}

\begin{tabular}{|llll|}
\hline \multicolumn{2}{|l|}{ SUBROUTINE } & sp2gp & \multicolumn{2}{l|}{ (sp, gp, lzm0) } \\
\hline name & type & intent & description \\
\hline mandatory & arguments: & & \\
sp & REAL(DP), DIMENSION $(:,:,:)$ & IN & spectral data \\
gp & REAL(DP), DIMENSION $(:,:,:)$ & OUT & grid-point data \\
lzm0 & LOGICAL & IN & wave number $\mathrm{m}=0 ?$ \\
\hline
\end{tabular}

This subroutine transforms data from (parallel decomposed) spectral space into (parallel decomposed) grid-point space. With $\operatorname{lzm} 0=$. TRUE. wave number $m=0$ is set to zero before transformation. 


\subsection{The subroutine gp2sp}

\begin{tabular}{|llll|}
\hline \multicolumn{2}{|l|}{ SUBROUTINE } & gp2sp & \multicolumn{2}{l|}{ (gp, sp, lzm0) } \\
\hline name & type & intent & description \\
\hline mandatory & arguments: & & \\
gp & REAL(DP), DIMENSION $(:,:,:)$ & IN & grid-point data \\
sp & REAL(DP), DIMENSION $(:,:,:)$ & OUT & spectral data \\
lzm0 & LOGICAL & IN & wave number $\mathrm{m}=0$ ? \\
\hline
\end{tabular}

This subroutine transforms data from (parallel decomposed) grid-point space into (parallel decomposed) spectral space. With $\operatorname{lzm} 0=$. TRUE. wave number $m=0$ is set to zero before transformation.

\subsection{The subroutine get_dc_index*}

\begin{tabular}{|llll|}
\hline \multicolumn{2}{|l|}{ SUBROUTINE } & get_dc_index & (N, IDX [, LDO] ) \\
\hline name & type & intent & description \\
\hline mandatory & arguments: & & \\
$\mathrm{N}$ & INTEGER & IN & global index range (number) \\
IDX & REAL(DP), DIMENSION(:,:) & index ranges per task \\
optional & arguments: & OUT & task is active? \\
LDO LOGICAL, DIMENSION(:) & OUT & \\
\hline
\end{tabular}

This subroutine calculates the index range (IDX $(i, 1: 2))$ of each task $i \in[0, n-1]$ for a parallel decomposition along an arbitrary index space with length $\mathrm{N}$. The optional output LDO(i) indicates, whether the resulting range of a specific task $i$ is zero (.FALSE.) or not (.TRUE.).

\subsection{The subroutine scatter_glix_1d*}

\begin{tabular}{|c|c|}
\hline SUBROUTINE scatter_glix_1d & $(\mathrm{gl}, \mathrm{lc}[, \mathrm{xpe}][, \mathrm{XNG}])$ \\
\hline name & description \\
\hline \multicolumn{2}{|l|}{ mandatory arguments: } \\
\hline REAL(DP), DIMENSION(:) & global data \\
\hline REAL(DP), DIMENSION(:) & decomposed (local) data \\
\hline \multicolumn{2}{|l|}{ optional arguments: } \\
\hline INTEGER & number of task with global data \\
\hline INTEGER & size of global data array \\
\hline
\end{tabular}

This subroutine scatters data of rank 1 as chunks onto the different tasks. The optional parameter xpe is for selecting a specific source task, which per default is the I/O task. The optional parameter XNG is to specify the length of the global data array gl. If this is a priori known, an internal broadcast of SIZE(gl) can be avoided.

\subsection{The subroutine scatter_glix_4d}

\begin{tabular}{|c|c|}
\hline SUBROUTINE scatter_glix_4d & $(\mathrm{gl}, \mathrm{lc}$, index $[, \mathrm{xpe}][, \mathrm{xishpg}])$ \\
\hline name & description \\
\hline \multicolumn{2}{|l|}{ mandatory arguments: } \\
\hline REAL(DP), DIMENSION(:,:,:,:) & global data \\
\hline REAL(DP), DIMENSION $(:,:,:,:)$ & decomposed (local) data \\
\hline INTEGER & along which rank? \\
\hline \multicolumn{2}{|l|}{ optional arguments: } \\
\hline INTEGER & number of task with global data \\
\hline INTEGER, DIMENSION(4) & shape of global data array \\
\hline
\end{tabular}

This subroutine scatters data of rank 4 as chunks along rank index onto the different tasks. The optional parameter xpe is for selecting a specific source task, which per default is the I/O task. The optional parameter xishpd is to specify the shape of the global data array gl. If this is a priori known, an internal broadcast of SHAPE(gl) can be avoided. 


\subsection{The subroutine gather_glix_1d*}

\begin{tabular}{|c|c|c|}
\hline SUBROUTINE gather_glix & \multicolumn{2}{|c|}{$(\mathrm{gl}, \mathrm{lc}[, \mathrm{xpe}][, \mathrm{XNG}])$} \\
\hline name & intent & description \\
\hline \multicolumn{3}{|l|}{ mandatory arguments: } \\
\hline REAL(DP), DIMENSION(:) & OUT & global data \\
\hline REAL(DP), DIMENSION(:) & IN & decomposed (local) data \\
\hline \multicolumn{3}{|l|}{ optional arguments: } \\
\hline INTEGER & IN & number of destination task \\
\hline INTEGER & IN & size of global data array \\
\hline
\end{tabular}

This subroutine gathers (or collects) decomposed data of rank 1 from all tasks into one global data array. The default destination task is the I/O task, but any other task can be selected by the optional parameter xpe. The optional parameter XNG is to specify the length of the global data array gl. If this is a priori known, an internal broadcast of SIZE (gl) can be avoided.

\subsection{The subroutine gather_glix_4d}

\begin{tabular}{|llll|}
\hline \multicolumn{2}{|l|}{ SUBROUTINE } & gather_glix & (gl, lc $[$, xpe $]$ [, XNG] $)$ \\
\hline name & type & intent & description \\
\hline mandatory & arguments: & & \\
gl & REAL(DP), DIMENSION $(:,:,:,:)$ & OUT & global data \\
lc & REAL(DP), DIMENSION $(:,,:,:)$ & IN & decomposed (local) data \\
index & INTEGER & IN & along which rank? \\
optional arguments: & & \\
xpe & INTEGER & IN & number of destination task \\
XNG & INTEGER & IN & size of global data array at rank index \\
\hline
\end{tabular}

This subroutine gathers (or collects) decomposed data of rank 4 from all tasks into one global data array. The default destination task is the I/O task, but any other task can be selected by the optional parameter xpe. The optional parameter XNG is to specify the size of the global data array gl at rank index. If this is a priori known, an internal summation of SIZE (lc, index) over all tasks can be avoided.

\section{Sub-submodel ATTILA_TOOLS (messy_attila_tools_e5)}

\subsection{The subroutine gp2lg_e5}

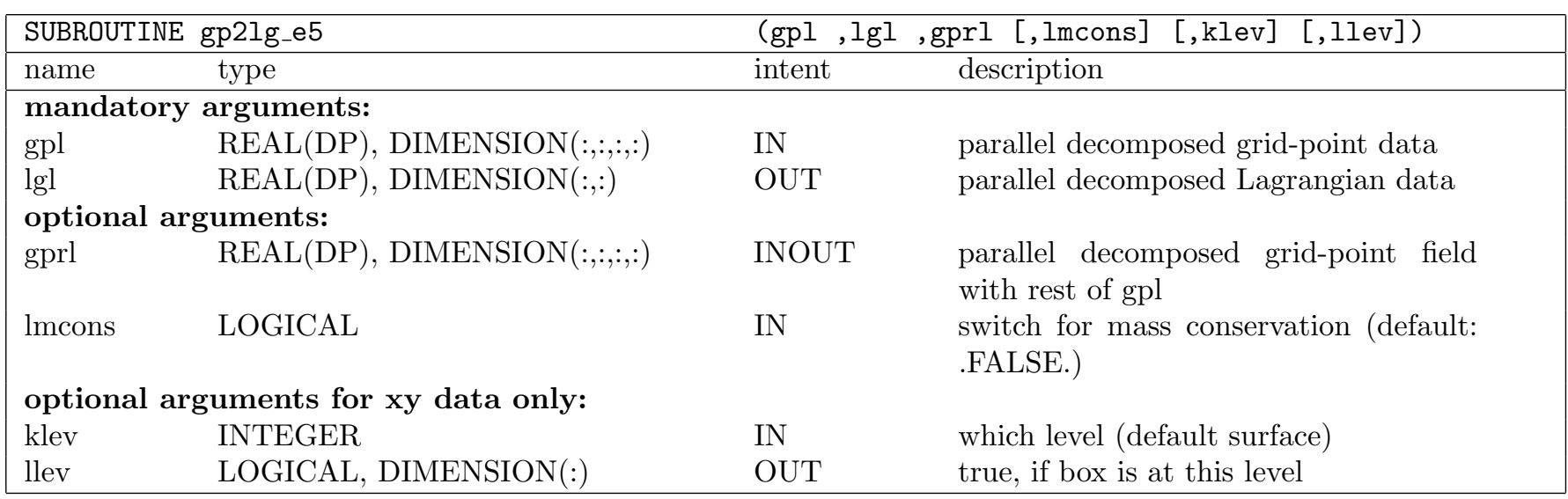

This subroutine performs the transformation of data from parallel decomposed grid-point space into parallel decomposed Lagrangian space. The optional argument Imcons has three different effects, depending on the presence of the grid-point variable gprl:

- Imcons=. FALSE. and . NOT . PRESENT (gprl):

The Lagrangian parcels receive the value of the grid-box corresponding to their locations, independent on other parcels located in the same grid-box. This means, all parcels in a specific grid-box get the same value. 
- Imcons=. TRUE. and .NOT . PRESENT (gprl):

The value of the grid-box is distributed as equal share among all Lagrangian parcels located in this grid-box. Information of grid-boxes, where currently no Lagrangian parcel is located, is lost.

- lmcons=. TRUE. and PRESENT (gprl):

The value of the grid-box is distributed as equal share among all Lagrangian parcels located in this grid-box. Information of grid-boxes, where currently no Lagrangian parcel is located, is stored and accumulated in gprl until the next Lagrangian parcel comes across.

This subroutine is threefold overloaded for rank 2 (xy), 3 (xyz) and 4 (xyzn, as shown above), respectively. Since gprl is used to conserve the total mass, gprl should be in $\mathrm{kg}$. However, the time-step length, the ratio of molar masses (air/tracer) etc. are scaling factors, at least as long as the time-step length does not vary during the integration. They are therefore omitted.

The subroutine for rank-2 data (xy) has two additional optional parameters: klev is used to select the Lagrangian parcels located at a specific grid-point level (default: surface level). The llev (.TRUE. or .FALSE.) returns whether a specific parcel is located in grid-point level $\mathrm{klev}$.

\subsection{The subroutine lg2gp_e5}

\begin{tabular}{|c|c|c|c|}
\hline \multicolumn{2}{|c|}{ SUBROUTINE lg2gp_e5 } & \multicolumn{2}{|c|}{$\begin{array}{l}\text { (lgl ,gpl ,method [,lmcons] [,ltm1] } \\
\text { [,fill_value] [,fill_field]) }\end{array}$} \\
\hline name & type & intent & description \\
\hline \multicolumn{4}{|c|}{ mandatory arguments: } \\
\hline $\lg l$ & REAL(DP), DIMENSION(:,:) & $\mathrm{IN}$ & parallel decomposed Lagrangian data \\
\hline & REAL(DP), DIMENSION(:,:,:,:) & OUT & parallel decomposed grid-point data \\
\hline method & INTEGER & $\mathrm{IN}$ & $\begin{array}{l}\text { LG2GP_AVE, LG2GP_SUM, } \\
\text { LG2GP_STD, LG2GP_AVEGT0 }\end{array}$ \\
\hline lmcons & LOGICAL & $\mathrm{IN}$ & $\begin{array}{l}\text { switch for mass conserving transforma- } \\
\text { tion (default .FALSE.) }\end{array}$ \\
\hline ltm1 & LOGICAL & IN & use positions of parcels at $t-\Delta t$ \\
\hline fill_value & REAL(DP) & $\mathrm{IN}$ & $\begin{array}{l}\text { optional value to be filled in, where no } \\
\text { parcel is located }\end{array}$ \\
\hline
\end{tabular}

This subroutine transforms data from Lagrangian space into grid-point space. A mass-conserving transformation can be requested with Imcons $=$. TRUE..

Four different methods (method) are possible:

- LG2GP_AVE: average over all parcels (on all tasks) in the corresponding grid-box,

- LG2GP_SUM: sum over all parcels (on all tasks) in the corresponding grid-box,

- LG2GP_STD: standard deviation over all parcels (on all tasks) in the corresponding grid-box,

- LG2GP_AVEGT0: average over all parcels (on all tasks) in the corresponding grid-box, where the value is $>0$.

Grid-boxes, where currently no parcel is located, are set to zero, or the fill_value, or the corresponding value from the fill_field. With the optional parameter ltm1 set to .TRUE., the parcel positions from the previous time step are used for the transformation (default: .FALSE., use actual positions).

This subroutine is twofold overloaded for data of rank 3 (xyz) and 4 (xyzn) as shown above, respectively. 


\subsection{The subroutine lggpte2lgte_e5}

\begin{tabular}{|c|c|c|c|}
\hline SUBROUTINE & lggpte2lgte_e5 & (gp , & lg, lgte) \\
\hline name & type & intent & description \\
\hline \multicolumn{4}{|c|}{ mandatory arguments: } \\
\hline gp & REAL(DP), DIMENSION(:,:,:,:) & IN & grid-point data \\
\hline gpte & REAL(DP), DIMENSION $(:,:,:,:)$ & IN & grid-point tendency \\
\hline $\lg$ & REAL(DP), DIMENSION(:,:) & IN & $\begin{array}{l}\text { Lagrangian data corresponding to gp } \\
\text { (above) }\end{array}$ \\
\hline lgte & REAL(DP), DIMENSION(:,:) & OUT & resulting Lagrangian tendency \\
\hline
\end{tabular}

This subroutine transforms a tendency from a grid-point process into a corresponding tendency in Lagrangian space. The grid-point field needs to be previously transformed from the corresponding Lagrangian data. A typical call sequence is:

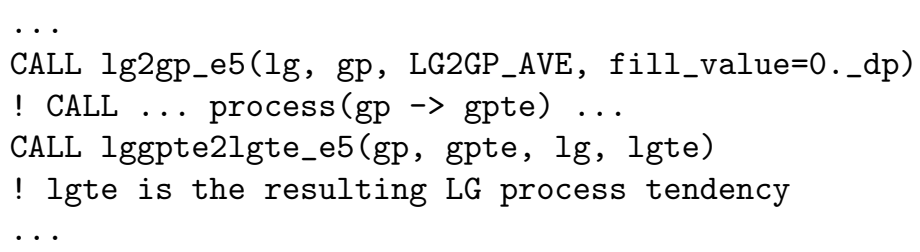

This subroutine is twofold overloaded for data of rank 3 (xyz) and 4 (xyzn) as shown above, respectively.

\subsection{The subroutine gpsfemis2lgemis_e5}

\begin{tabular}{|c|c|c|c|}
\hline \multicolumn{2}{|c|}{ SUBROUTINE gpsfemis2lgemis_e5 } & \multicolumn{2}{|c|}{ (gpl, lgl, method [,gprl] [,lmcons]) } \\
\hline name & type & intent & description \\
\hline \multicolumn{4}{|c|}{ mandatory arguments: } \\
\hline gpl & REAL(DP), DIMENSION(:,:) & IN & global grid-point data \\
\hline $\operatorname{lgl}$ & REAL(DP), DIMENSION(:) & OUT & Lagrangian data \\
\hline method & INTEGER & IN & $1,2,3$ or 4 \\
\hline \multicolumn{4}{|c|}{ optional arguments: } \\
\hline gprl & REAL, DIMENSION(:,:) & INOUT & $\begin{array}{l}\text { grid-point array with rest of mass (if no } \\
\text { parcel resides in grid box) }\end{array}$ \\
\hline $\operatorname{lmcons}$ & LOGICAL & IN & $\begin{array}{l}\text { switch for mass conserving transforma- } \\
\text { tion }\end{array}$ \\
\hline
\end{tabular}

This subroutine transforms grid-point surface emission fluxes (any intensive quantity per unit time, e.g. mol $/ \mathrm{mol} / \mathrm{s}$, $\mathrm{kg} / \mathrm{kg} / \mathrm{s}, \mathrm{mol} / \mathrm{m}^{2} / \mathrm{s}$ etc.) into corresponding Lagrangian fluxes (in the same units). Mass conserving transformation can be requested with the optional parameter lmcons set to .TRUE. (default: .FALSE.). Depending on the presence of gprl, the fluxes into grid boxes, where no parcels are located, are either lost, or stored in gprl and accumulated until the next parcel comes across.

Four different emission methods (method) are implemented: the emission flux is distributed among all parcels

1. in the surface layer,

2. located lowest in the boundary layer,

3. in the boundary layer with a negative vertical gradient,

4. in the boundary layer with equal share.

\section{ATTILA namelists}

The user interface of the submodel ATTILA is the namelist file attila.nml. It contains the control namelist \&CTRL (see Fig. 1), the coupling namelist \&CPL (see Fig. 2) and the namelist \&TRAJ (see Fig. 3) for the so-called trajectory mode. The user can specify in the control namelist \&CTRL: 
- NCHUNK (default: 48): the number of chunks into which loops over all parcels are decomposed. This can be used for hybrid parallelization (e.g., OpenMP), however, is currently not implemented.

- CPGBAVE (default: 2.2): the average number of parcels per grid-box. From this average number, the total number of parcels is determined, if I_NCELL $<0$ (see below).

- LLTINFO (default: .FALSE.): output of additional information into log-file.

- I_PBLH_METHOD (default: 0): method to calculate the planetary boundary layer height (and with that the first layer in the free troposphere); options are 0 (calculation within ATTILA) or 1 (external calculation).

- ADICO specifies the diffusion coefficients for the Monte Carlo diffusion:

- ADICO (1) is the horizontal diffusion coefficient for the free atmosphere in $\mathrm{m}^{2} \mathrm{~s}^{-1}$,

- ADICO (2) is the horizontal diffusion coefficient for the boundary layer in $\mathrm{m}^{2} \mathrm{~s}^{-2}$, and

$-\operatorname{ADICO}(3)$ is the vertical diffusion coefficient in $\mathrm{s}^{-1}$.

If $\operatorname{ADICO}(:)==0.0$ any Monte Carlo diffusion is omitted (see Sect. 2.2.1 in main text).

- LLTBLTURB (default: .FALSE.) is the switch for the boundary layer turbulence: If LLTBLTURB=. TRUE., Lagrangian parcels are randomly displaced within the boundary layer by Monte Carlo diffusion.

- LLCONV (default: .FALSE.) is the switch for the convective movement of parcels.

- LLCAT (default: .FALSE.) is the switch for the turbulent movement of parcels in clear air turbulence areas. This is, however, currently not implemented.

- LVDIAG (default: .FALSE.) is a switch for additional velocity diagnostics.

- I_NCELL (default: -1) sets the global number of parcels: If I_NCELL $<0$, the number is calculated from the number of grid-boxes times the average number of particles (CPGBAVE, see above), i.e., depending on the gridpoint resolution. For I_NCELL $>=0$ the number is set directly.

- LTRAJEC (default: .FALSE.) is to select the so-called trajectory mode, which allows to release individual parcels at given times and locations. If this mode is selected (.TRUE.),

- LTRAJEC_SAME_DATE (default: .FALSE.) can be used to overwrite the individual start dates and times (see \&TRAJ namelist below) with the same date and time specified by

- LTRAJEC_DATE (default: 1978, 1, 1, 1, 0) in the from of Y, M, D, HR, MI, where Y, M, D, HR, MI denote year, month, day, hour and minute (UTC), respectively.

- I_VERT (default: 1) selects the vertical coordinate system and the corresponding vertical velocity scheme (see Sect. 2.2.2 in main text):

1. eta-coordinate system,

2. theta-sigma hybrid coordinate system,

3. sigma-coordinate system.

- press_ref in $\mathrm{Pa}$ is the reference pressure, for I_VERT=2, where the theta-sigma hybrid coordinate system changes to a pure theta-coordinate in the stratosphere. If press_ref $=-1$, the reference pressure level is the tropopause level.

In the \&CPL namelist of ATTILA the user can specify:

- L_INI_PARALLEL (default: .TRUE.) to perform the initialization of parcel positions in parallel mode.

- I_RANDOM_METHOD (default: 0) to select the pseudo-random number generation method (see Sect. 2.2.1 in main text):

- 0: Fortran intrinsic,

- 1: Mersenne Twister, 


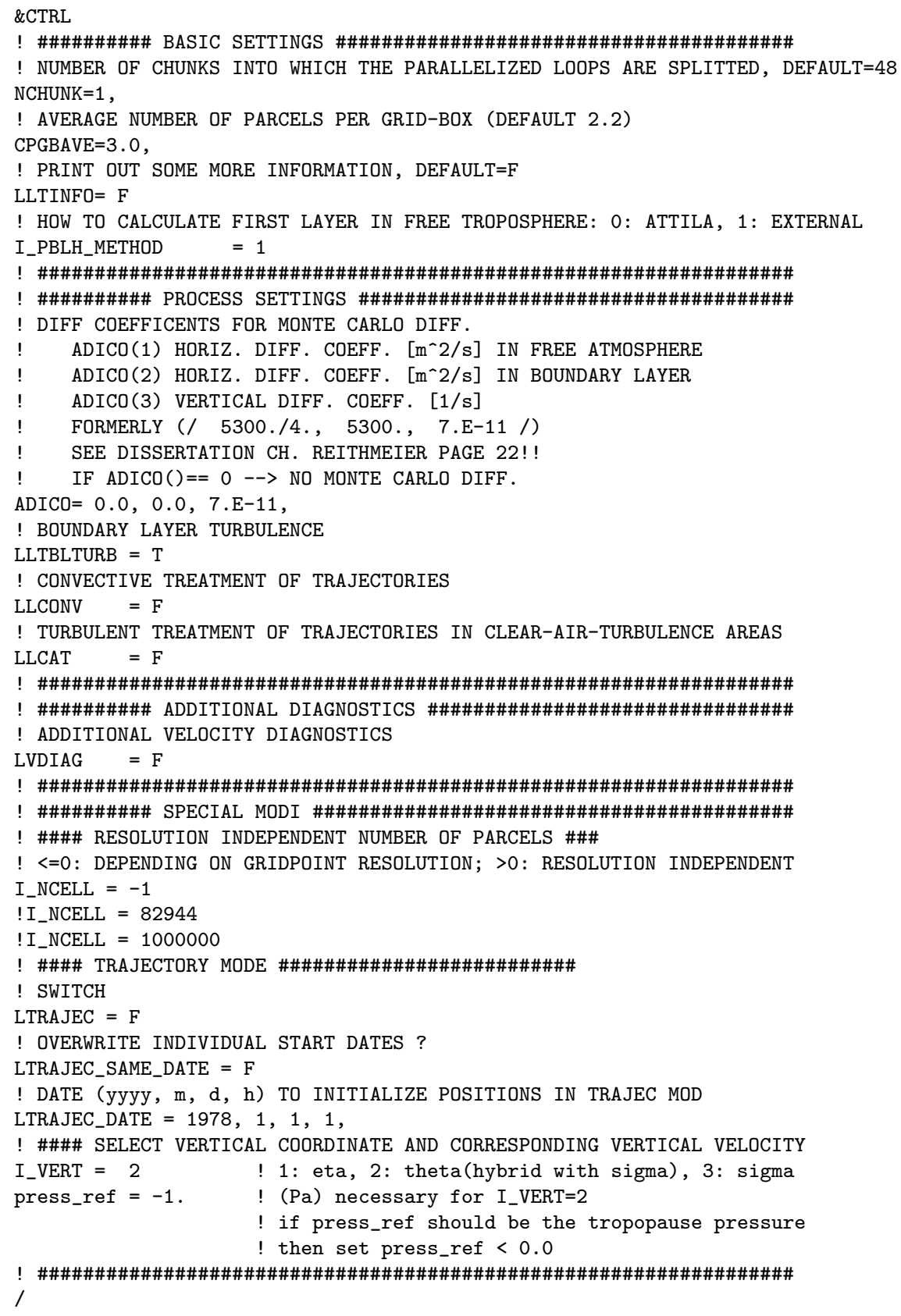

Figure 1: Example \&CTRL namelist of the submodel ATTILA.

-2 : Luxury.

- I_RANDOM_PARALLEL (default: 3) to select the parallel generation of pseudo-random numbers:

- 3: parallel synchronized by jumping ahead,

- 4: independent by jumping ahead.

- L_RANDOM_TEST (default: .FALSE.) to create an additional channel object to output random numbers for testing.

- the channel and channel object names used to drive ATTILA:

- C_PBLH_INDEX to select the externally calculated level index of the planetary boundary layer height (if I_PBLH_METHOD $=1$, see above in \&CTRL namelist). 
- C_CONV_UFLX, C_CONV_DFLX and C_CONV_TYPE to select the updraft mass flux, the downdraft mass flux, and the type of convection, respectively (if LLCONV = T, see above in \&CTRL namelist).

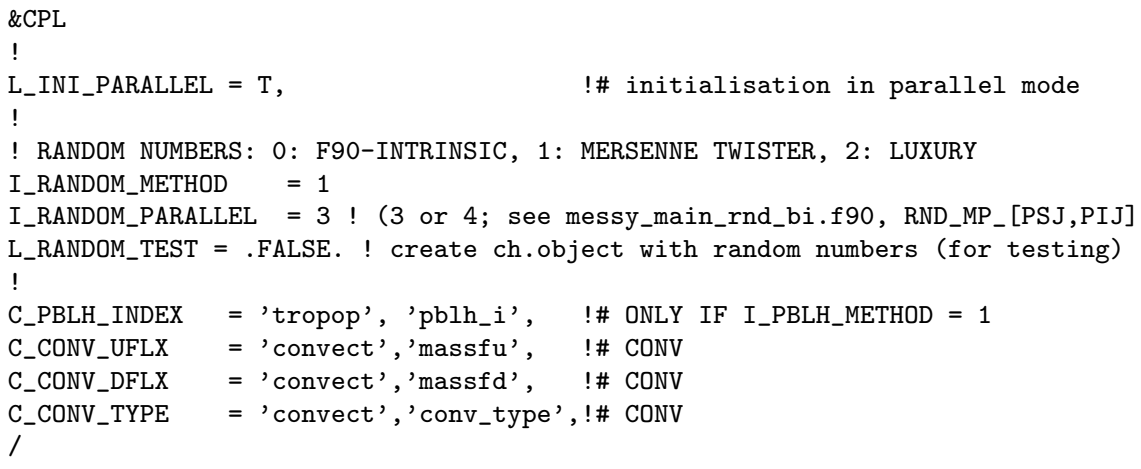

Figure 2: Example \&CPL namelist of the submodel ATTILA.

The \&TRAJ namelist controls the release locations and dates/times of individual parcels in the so-called trajectory mode (if LTRAJEC=. TRUE. in the \&CTRL namelist). These are specified as ATTILA Parcels (with keyword AP) with an arbitrary but unique number, with position (latitude, longitude and pressure altitude (in Pa)), release date (year, month, time) and release time (hour, minute (UTC)).

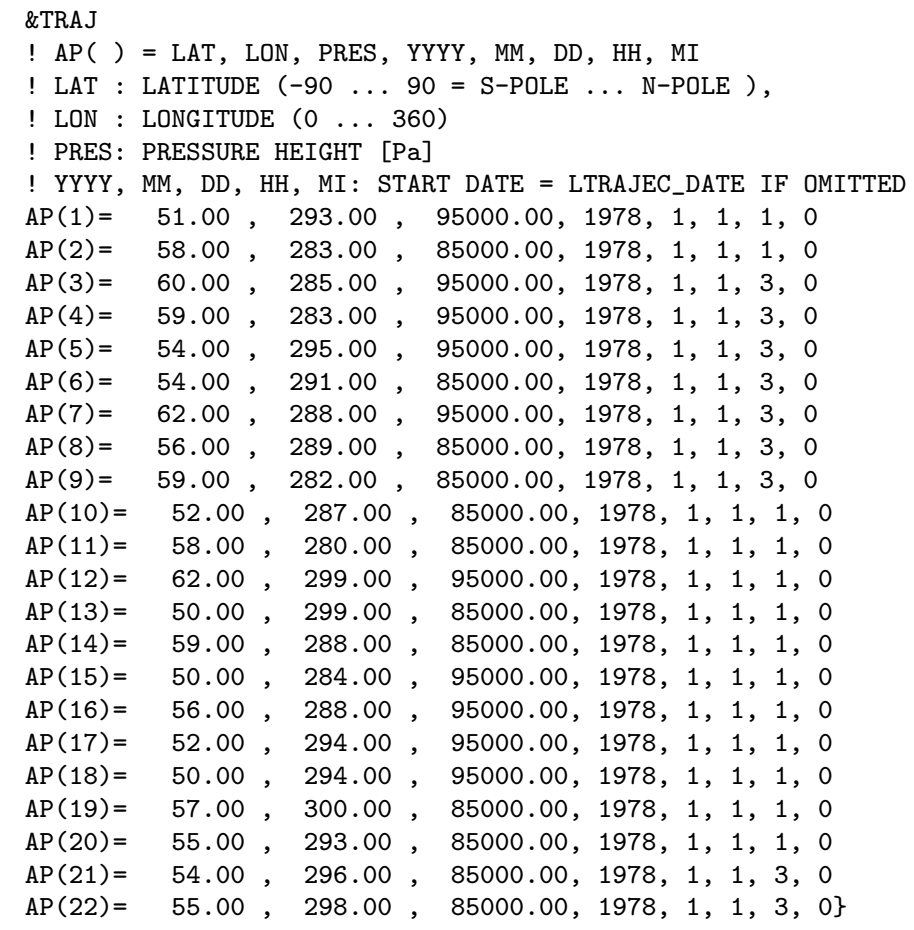

Figure 3: Example \&TRAJ namelist of the submodel ATTILA. 


\section{$5 \quad$ LGGP namelist}

The LGGP coupling namelist \&CPL (example see Fig. 4) allows to define new objects for output, which are either transformed from Lagrangian space into grid-point space (LG2GP) or from grid-point space to Lagrangian space (GP2LG). This renders any hard-wired coding of variable transformations unnecessary, see also Sect. 3. For LG2GP one need to specify:

- the keyword LG2GP with an arbitrary but unique number in parentheses,

- the name of the Lagrangian channel containing the object to be transformed,

- the object name representing the Lagrangian variable to be transformed,

- the transformation method:

- 1: sums up the properties of the parcels in each grid-box,

- 2: averages the properties of the parcels in each grid-box,

- 3: calculates the standard deviation of the properties of the parcels in each grid-box.

- a logical switch to determine, if the transformation should be mass conserving (.TRUE.) or not (.FALSE.),

- the switch fill_flag to select, how grid-boxes, which contain no parcels, should be filled:

- 0: no filling (filling with zero)

- 1: a single fill value fill_value

- 2: filling with (the corresponding grid-box value of) a grid-point field.

- the value fill_value for fill_flag=1,

- the channel and the channel object name of the grid-pint field, if fill_flag=2.

For GP2LG one need to specify:

- the keyword GP2LG with an arbitrary but unique number in parentheses,

- the name of the grid-point channel containing the object to be transformed,

- the object name representing the grid-point variable to be transformed,

- a logical switch to determine, if the transformation should be mass conserving (.TRUE.) or not (.FALSE.),

- a logical switch to account for information in grid-boxes, where currently no Lagrangian parcel is located; if .TRUE., this information is stored and accumulated until the next Lagrangian parcel comes across. 


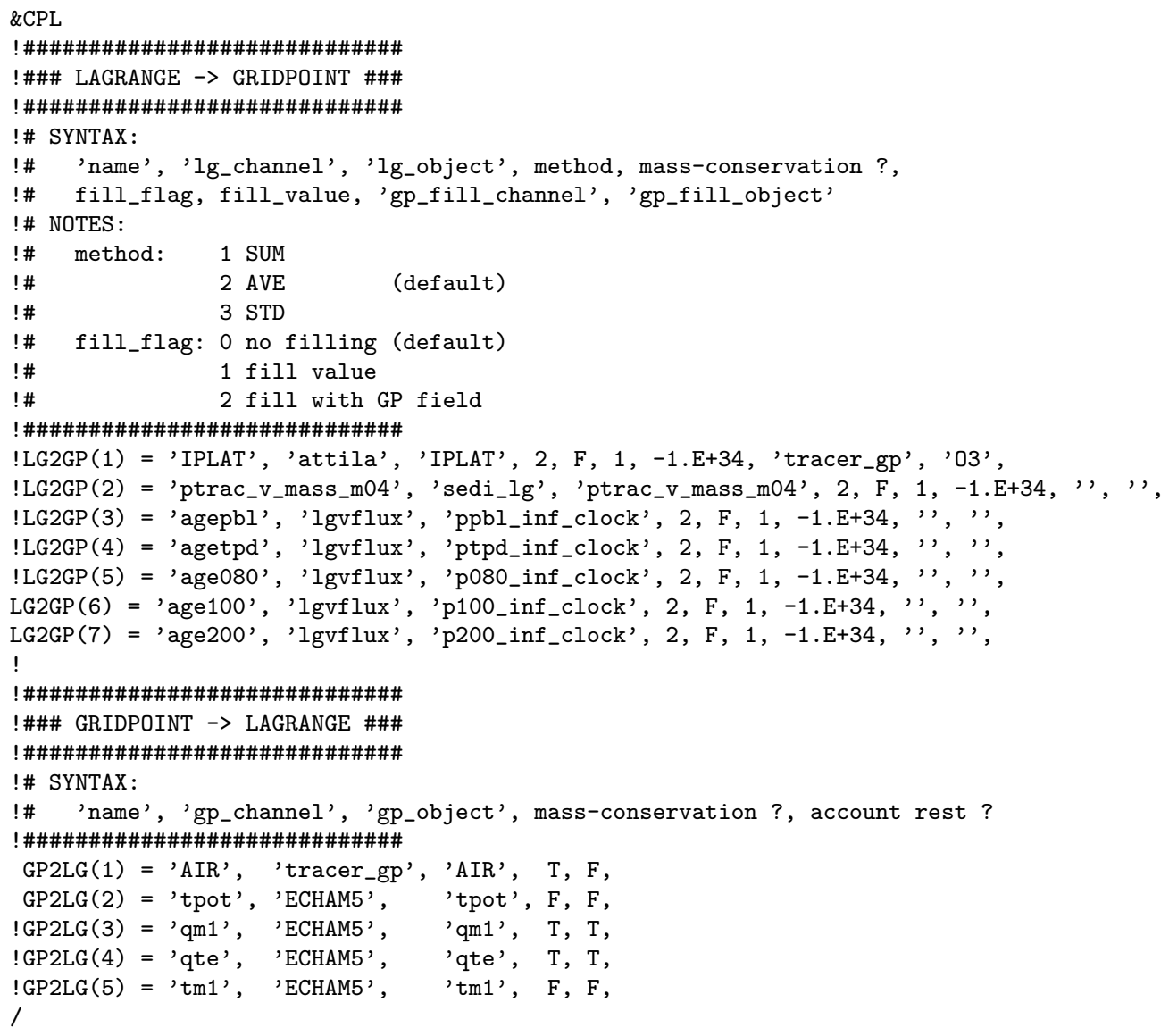

Figure 4: Example \&CPL namelist of the submodel LGGP

\section{LGVFLUX namelist}

LGVFLUX is a diagnostic submodel, which calculates on-line vertical fluxes of Lagrangian parcels through horizontal surfaces. The selected horizontal surfaces and flux-quantities must be specified in the LGVFLUX \&CPL namelist (example see Fig. 5). Horizontal surfaces can for instance be defined with the submodel VISO (Jöckel et al., 2010), e.g., isentropes, pressure levels, levels of constant potential vorticity, etc.. The LGVFLUX \&CPL namelist contains:

- the keyword VDYN with an arbitrary but unique number in parentheses (example vdyn(1)), with

- a unique channel object name (p080_096 in the example),

- the channel name containing the horizontal surface in grid-point space (viso in the example),

- the channel object name of the horizontal surface (2d) in grid-point space (pp080 in the example), and

- the minimum residence time to account for the flux after the parcels transition through the selected surface (345600.0 $\mathrm{s}$ in the example).

- the keyword VFLX with an arbitrary but unique number in parentheses (example vflx(1)), with

- a unique channel object name of the vertical flux quantity (air_p080_096 in the example),

- the channel object name of the corresponding VDYN object (see above),

- the channel name containing the object of which the flux should be calculated,

- the channel object name of which the flux should be calculated, and

- optionally the channel name and channel object name of the corresponding tendency, in case it should be taken into account for prognostic variables (e.g., tracers). 


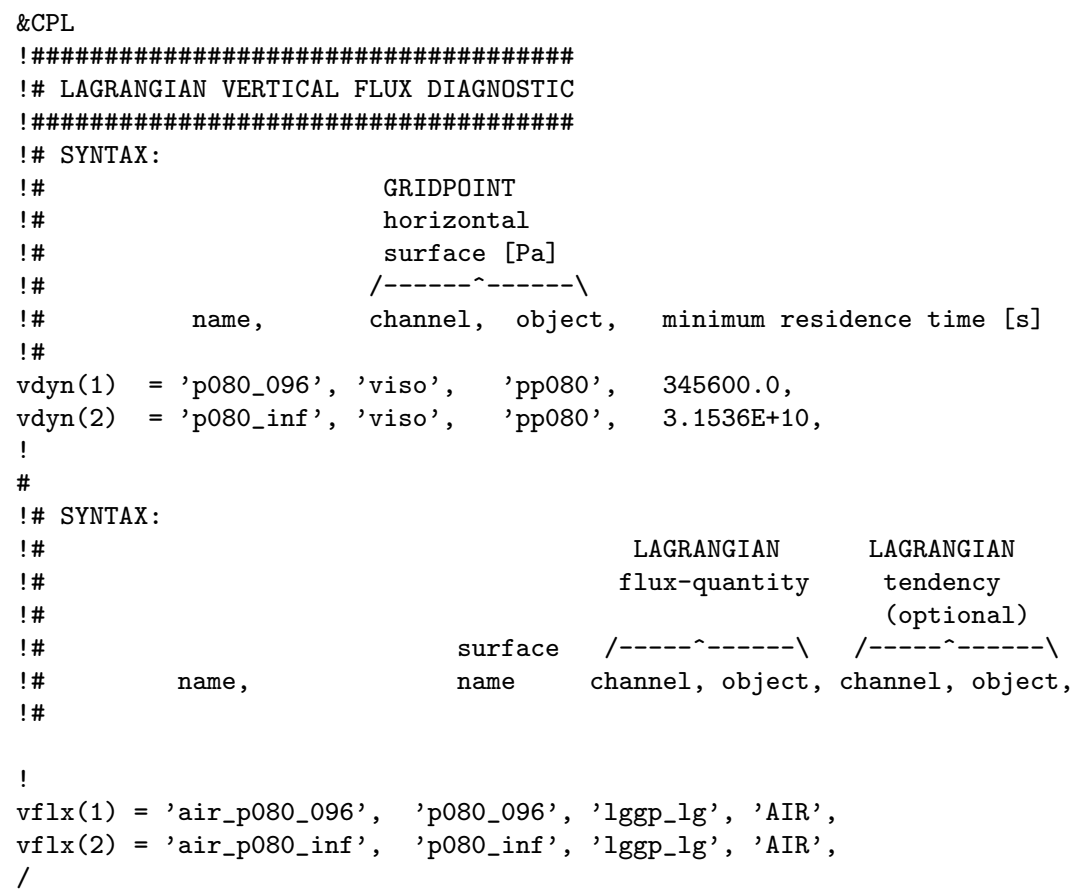

Figure 5: Example \&CPL namelist of the submodel LGVFLUX

\section{LGTMIX namelist}

The submodel LGTMIX describes the inter-parcel mixing as a process, where each parcel in a grid-box communicates with the background concentration (the mean over all parcels in that grid-box). The mixing parameter for different regions (layers) of the atmosphere can be specified in the \&CPL namelist (example see Fig. 6):

- With I_force=.TRUE., the 3d mixing parameter will be defined as a channel object, e.g., for output.

- A mixing layer is specified with the keyword MX and an arbitrary but unique number in parentheses; it is defined by specifying

- the lower bound of the vertical (level index) range as a channel object with channel name (e.g., tropop) and channel object name of the vertical level index (e.g., pbh_i),

- the upper bound of the vertical (level index) range as a channel object with channel name (e.g., tropop) and channel object name of the vertical level index (e.g., tp_i),

- the mixing parameter (see Sect. 2.4 in the main text), either

* by a constant, or

* by a channel object (with channel name and channel object name) and a corresponding value range to be mapped onto the interval $[0,1]$.

- With the keyword TX (and an arbitrary, but unique number in parentheses), the mixing parameter can be scaled additionally for each tracer individually; this is defined by (see Jöckel et al., 2008, for details on tracers)

- the basename of the tracer,

- the subname of the tracer, and

- a list of scaling factors (one for each layer defined by MX). 


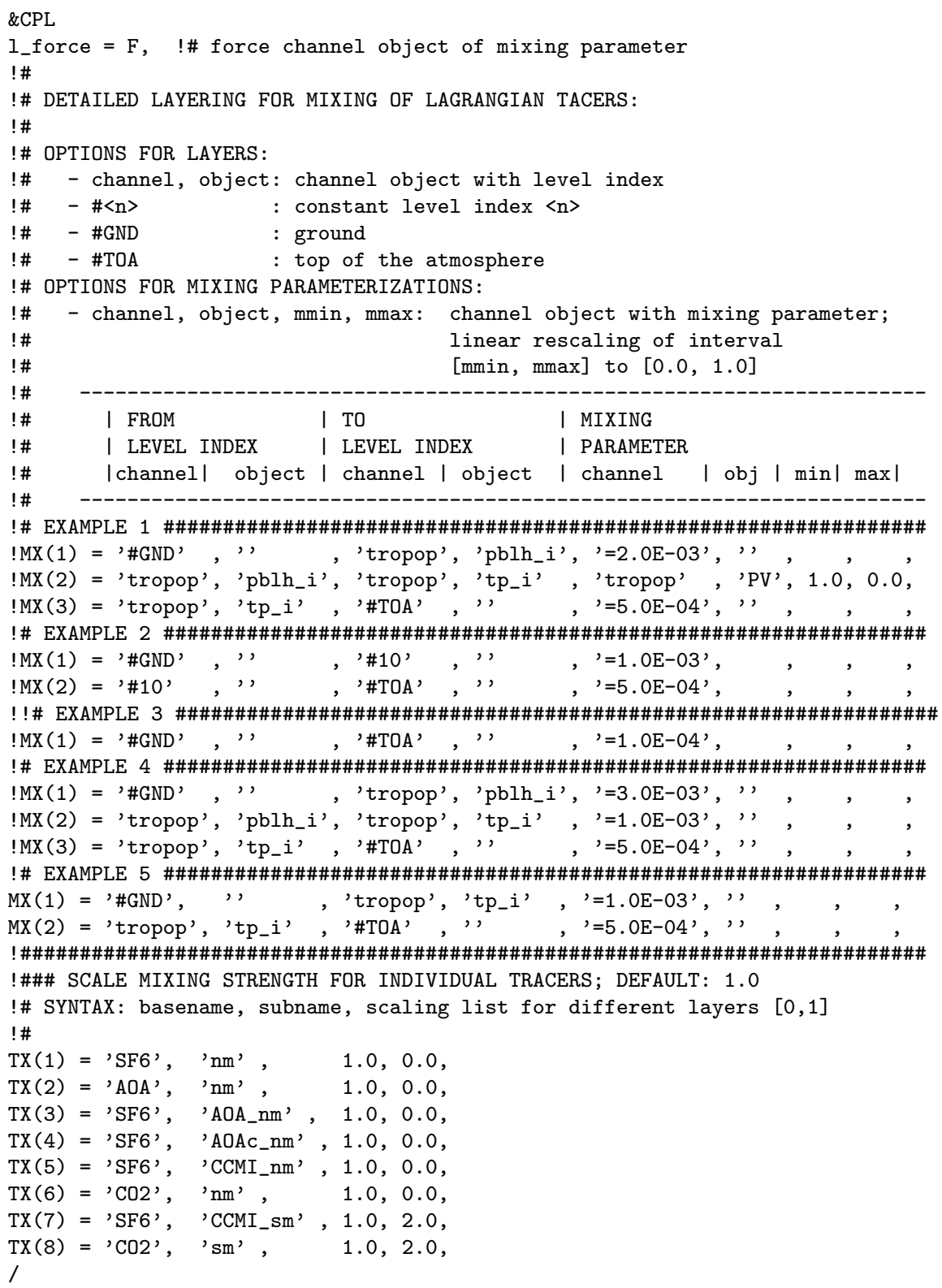

Figure 6: Example \&CPL namelist of the submodel LGTMIX.

\section{DRADON namelist extension}

The DRADON submodel and the corresponding namelists are described by Jöckel et al. (2010). For the application with ATTILA, the submodel has been extended. New features can be set in the \&CPL namelist of DRADON (example see Fig. 7):

- L_LG is used to switch the Lagrangian calculations on (.TRUE.) or off (.FALSE.).

- I_LG_emis_method selects the treatment of the ${ }^{222} \mathrm{Rn}$ emissions (see Sect. 3.4):

- 1: emission into parcels in the lowest model layer,

- 2: emission into parcels located lowest in the boundary layer,

- 3: emission into parcels located in the boundary layer with negative vertical gradient,

- 4: emission into parcels located in the boundary layer. 
- L_LG_emis_mcons is the logical switch for mass conserving transformation of the grid-point emission.

- I_LG_emis_rest determines how the emissions from grid-boxes, where currently no parcel is located, are treated:

--1 (automatic, default): The accounting of the rest is detemined automatically from I_LG_emis_method:

$*: 1$ : on,

* : 2,3,4: off.

- 0 (off): The flux is unaccounted.

-1 (on): The flux is stored and accumulated until the next parcel comes across.

- L_LG_emis_rest_int determines, if the accumulated rest flux from boxes (where no parcel is located) decays (.TRUE.) or not (.FALSE.).

- $\mathrm{L} \_L G_{-}$chain selects, if only the ${ }^{222} \mathrm{Rn}$ decay is simulated (.FALSE.), or the decay chain up to ${ }^{210} \mathrm{~Pb}$ (.TRUE.).

- If the decay chain is simulated (L_LG_chain = .TRUE.), C_LG_210Pb_aermod and I_LG_210Pb_mode set the aerosol model and mode number for the treatment of the ${ }^{210} \mathrm{~Pb}$ tracer, respectively. From these, the corresponding aerosol mean radius and aerosol radius standard deviation are used as tracer properties of ${ }^{210} \mathrm{~Pb}$ (for sedimentation and scavenging).

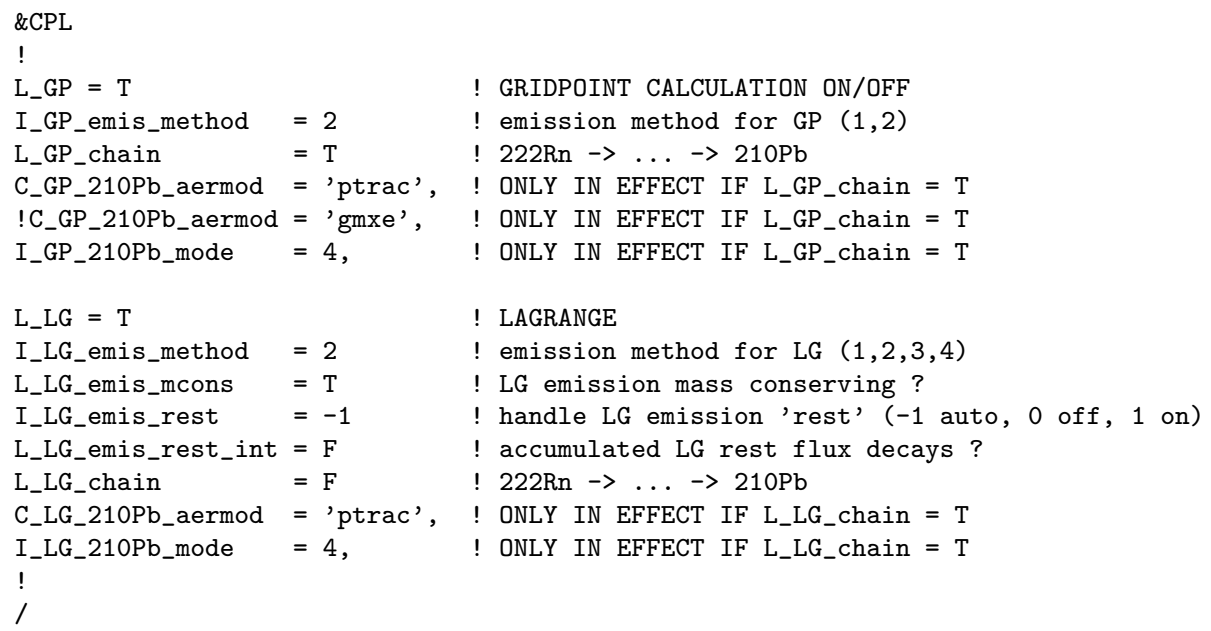

Figure 7: Example \&CPL namelist of the submodel DRADON with the Lagrangian extension.

\section{References}

Jöckel, P., Kerkweg, A., Buchholz-Dietsch, J., Tost, H., Sander, R., Pozzer, A., Technical Note: Coupling of chemical processes with the Modular Earth Submodel System (MESSy) submodel TRACER, Atmos. Chem. Phys., 8, No 6, 1677-1687,http://www.atmos-chem-phys.net/8/1677/2008/,10.5194/acp-8-1677-2008, 2008.

Jöckel, P., A. Kerkweg, Pozzer, A., Sander, R., Tost, H., Riede, H., Baumgaertner, A., Gromov, S., and Kern, B.: Development cycle 2 of the Modular Earth Submodel System (MESSy2), Geosci. Model Dev., 3, 717-752, doi:10.5194/gmd-3-717-2010, 2010. 